\title{
Transdiaphragmatic Liver Hernia in Adults
}

\author{
FAROOQ P. AGHA, M.D. \\ Division of Abdominal Radiology, \\ Department of Radiology, University Hospital, \\ University of Michigan Medical Centre, Ann Arbor, MI 48109.
}

\section{INTRODUCTION}

Right sided diaphragmatic hernia exclusive of the hiatus is uncommon. It is probably due to the earlier closure of the right pleuroperitoneal foramen and protection of the foramen afforded by the liver!. Hepatic herniation through the right leaf of the diaphragm, though still rare, is being reported with increasing frequency.

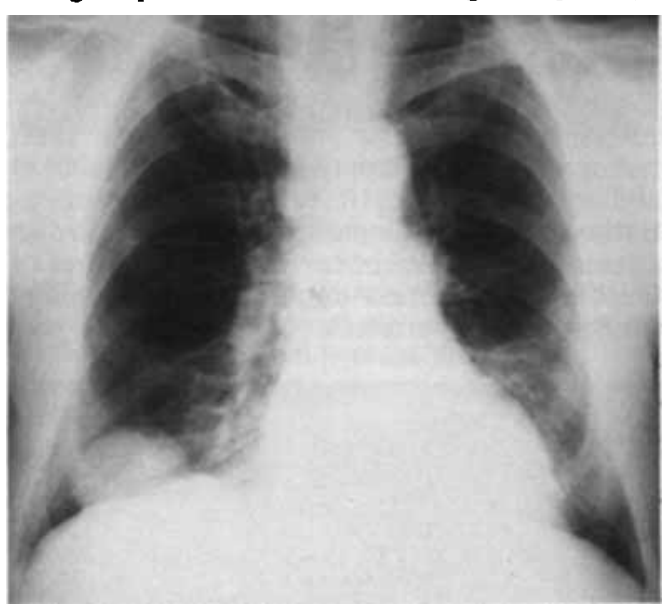

FIGURE IA

FIGURE 1A and FIGURE IB. - Case I: PA and lateral views of the chest showing a $4 \times 5 \mathrm{cms}$. smooth oval shaped mass in the right lower lung field with its inferior border abutting against the diaphragm.

\section{Keywords:}

Hepatic Herniation, Hernia. Hepatic, Liver Intrathoracic, Diaphragmatic Hernia.

Address reprint requests to:

Farooq P. Agha, M.D.

Department of Radiology,

Box 13 ,

University Hospital.

Ann Arbor, MI 48109

Telephone: (313) 764-0285.
Hedblom $^{2}$, in a review of 857 cases of diaphragmatic hernias, found 72 different combinations of herniations of the stomach, small bowel, colon, omentum, spleen, liver, pancreas and the kidney. Herniation of the liver alone was found in 14 cases. Liver hernias may be congenital or acquired. The congenital diaphragmatic hernias containing portion of the liver usually occur through the foramen of Bockdalek $^{3,4}$, the foramen of Morgagni ${ }^{5}$, the esophageal hiatus ${ }^{6}$ and, very rarely, through a defect left by partial absence of the diaphragm? . All acquired transdiaphragmatic liver hernias are post traumatic. According to the clinical presentation, traumatic rupture of the diaphragm can be divided into acute, latent and obstructive phases $^{8}$. The asymptomatic latent phase may range from 3 months to 44 years 9.10 .

Three previously unreported cases of transdiaphragmatic hepatic herniation in adults developing $10-20$ years after the initial history of trauma have prompted this review.

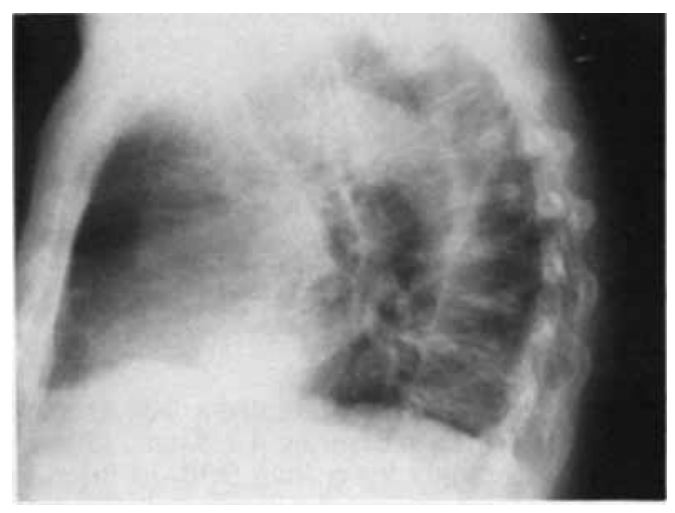

FIGURE 1B

Accepted for publication on 4 th October, 1984. 

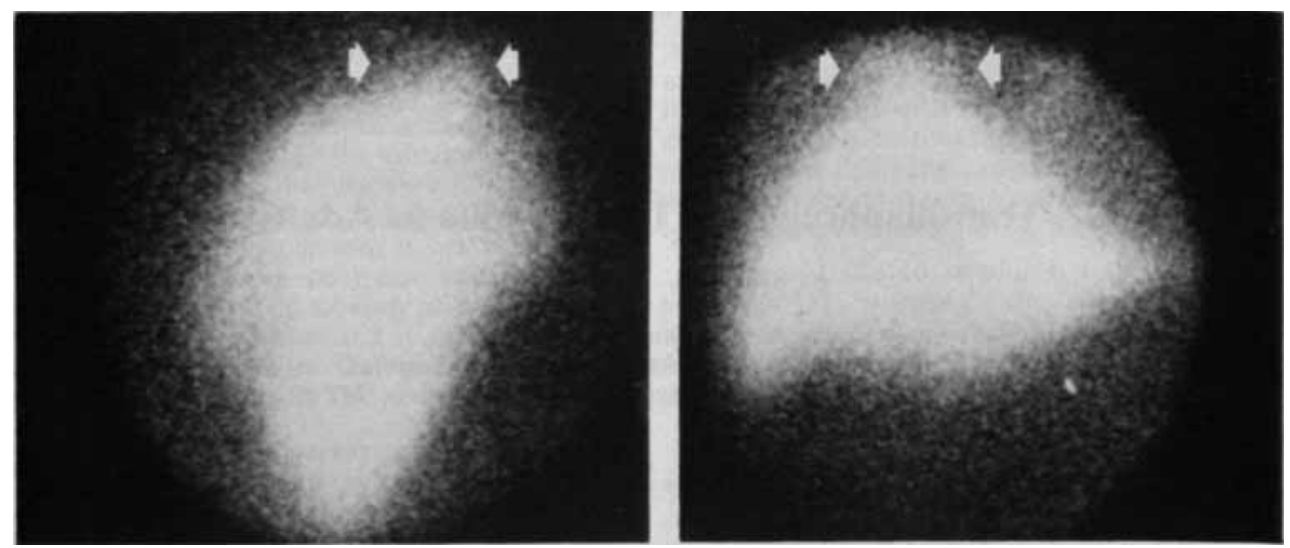

FIGURE IC and FIGURE 1D. - Case I: Radionuclide liver scan in anterior and right lateral projections revealing an abnormal bulge along the anterior and superior aspect of the right lobe of the liver (arrows).

\section{CASE REPORTS}

Case 1:

This 85-year old white male was admitted to Veterans Administration Medical Centre, Ann Arbor on $12 / 17 / 79$ for evaluation of a right sided lung mass and back pain. An abnormal lung mass was noted on chest radiograph obtained a week earlier during investigation for upper respiratory infection. He had smoked $1 / 3$ of a package of cigarettes per day until approximately 15 years previously. His low back pain dated back to a motor vehicle accident in 1965 . There had been a recent increase in severity of the low back pain with radiation to the buttocks. His significant past history consisted of a mild rightsided cerebrovascular accident in 1978 and a motor vehicle accident in 1965 in which he sustained a right hip fracture, and a fracture of the $L 1$ vertebral body. He did not sustain any internal injuries. The hip fracture was treated by open reduction and internal fixation. The physical examination revealed a well developed male in no acute distress. Vital signs showed a blood pressure of $90 / 150$, pulse $80 /$ minute and regular, respiration rate of 16/minute. Except for a grade 11/IV ejection systolic murmur the physical examination was unremarkable. The laboratory data revealed normal hematological and biochemical profiles except for serum glucose of $184 \mathrm{mg} / 100 \mathrm{ml}$.

The chest radiograph revealed a well circumscribed oval mass measuring $4 \times 5 \mathrm{cms}$. in size, located in the right lower lung field, its inferior border abutting against the diaphragm (Figures $1 \mathrm{~A}$ and 1B). Previous radiographs of the chest in 1975 revealed similar findings. It was felt that a neoplasm could not be ruled out, because in the patient's age group neoplasia may have a very slow growth rate. Therefore, further work-up was initiated. A radionuclide liver scan (Figures IC and 1D) revealed an abnormal bulge along the anterior and superior aspect of the right lobe corresponding to the mass seen in the chest radiograph. A CT scan (Figure 1E) of the thorax and upper abdomen revealed a soft tissue intrathoracic Juxta-diaphragmatic mass with an attenuation coefficient corresponding to that of the liver. Ultrasound examination (Figure 1F) confirmed the presence of a defect in the

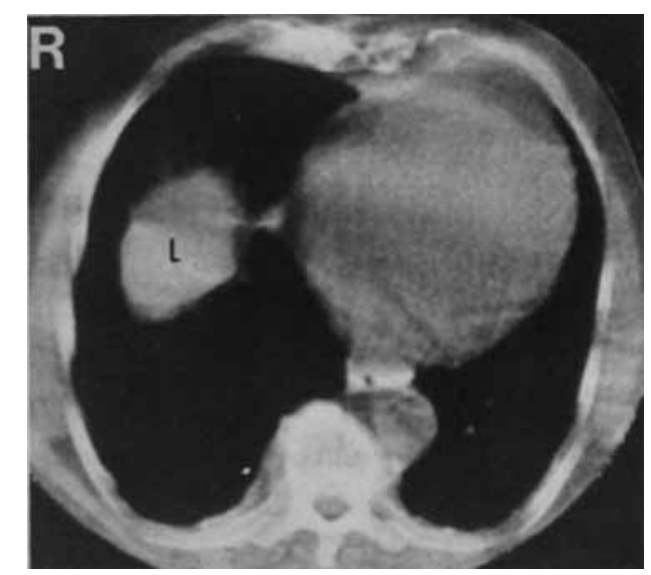

FIGURE 1E. - Case 1 : A CT scan cut $3 \mathrm{~cm}$. above the diaphragmatic level showing the intrathoracic supradiaphragmatic mass having the same attenuation coefficient as that of liver.

$\mathbf{L}=$ Liver.

Australasian Radiology, Vol. XXIX, No. I, February, 1985 


\section{TRANSDIAPHRAGMATIC LIVER HERNIA IN ADULTS}

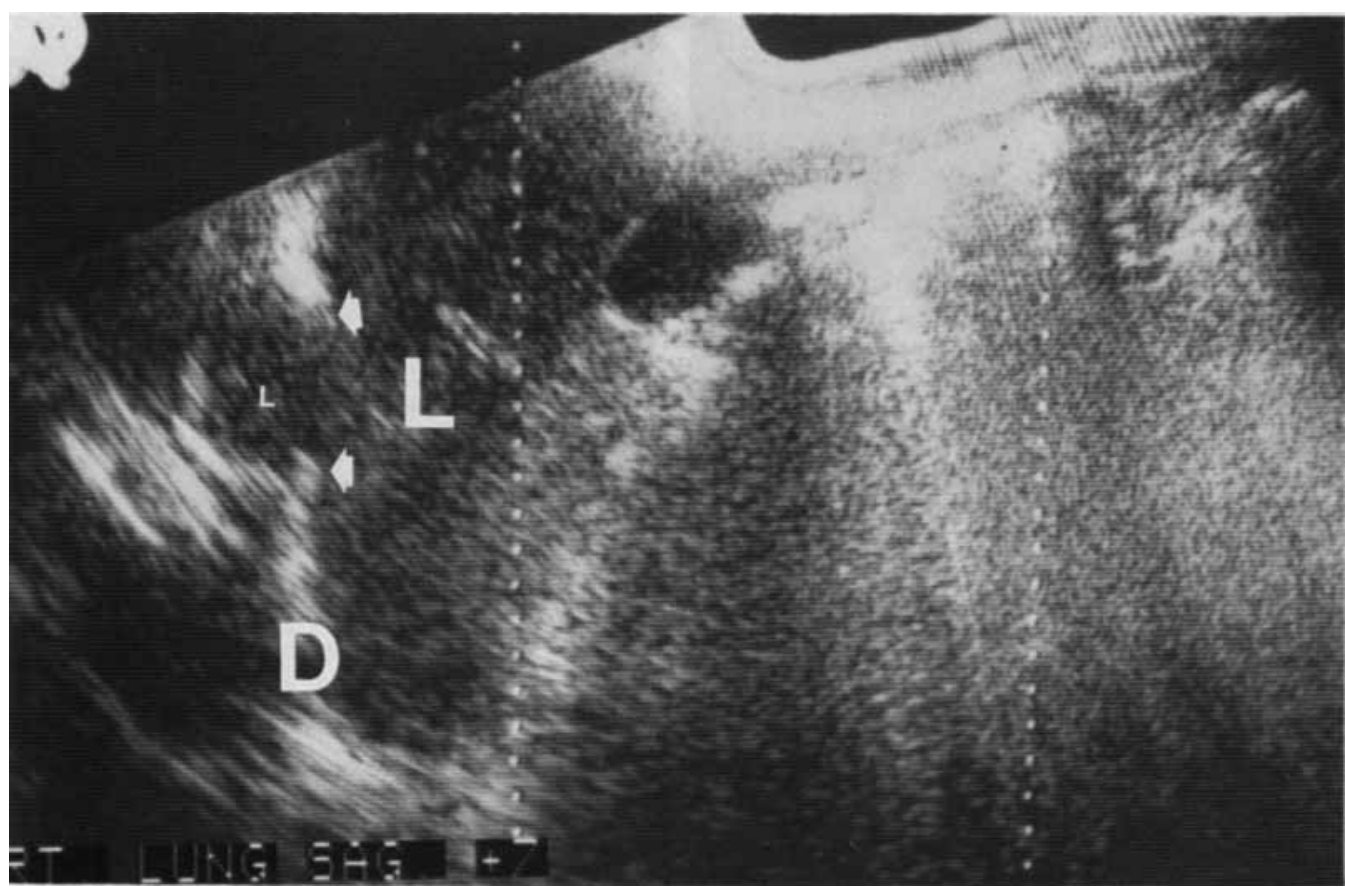

FIGURE 1F. - Case I: Longitudinal ultrasound scan through the right upper quadrant of the abdomen showing a defect (arrows) in the diaphragm with liver tissue protruding into the thorax.

$\mathbf{L}=$ Liver, $\mathbf{D}=$ Diaphragm.

diaphragm with liver tissue protruding into the thorax. Since the patient was asymptomatic no treatment was deemed necessary.

His lumbosacral spine $\mathrm{x}$-rays revealed an old compression fracture of the L1 vertebra. Symptomatic treatment with pain medication, bedrest and heat pads were recommended. The patient was last seen in June 1982. The chest findings, representing herniated liver, remained unchanged.

\section{Case 2:}

This 66-year old white male was admitted to the Veterans Administration Hospital, Ann Arbor on 8/16/65 for evaluation of an abnormal chest radiograph. He complained of periodic mild dyspnea and a dry cough. He had recently developed vague abdominal pain and nausea. He had been an occasional cigarette smoker all his life.

The past history revealed an auto accident 20 years previously in which he had sustained bilateral rib and pelvic bone fractures but no apparent internal injuries. Physical examination revealed a well nourished white male in no acute distress. Examination of the chest revealed a slight increase in its anterior posterior diameter. Breath sounds were decreased over the right lower hemithorax. Questionable bowel sounds

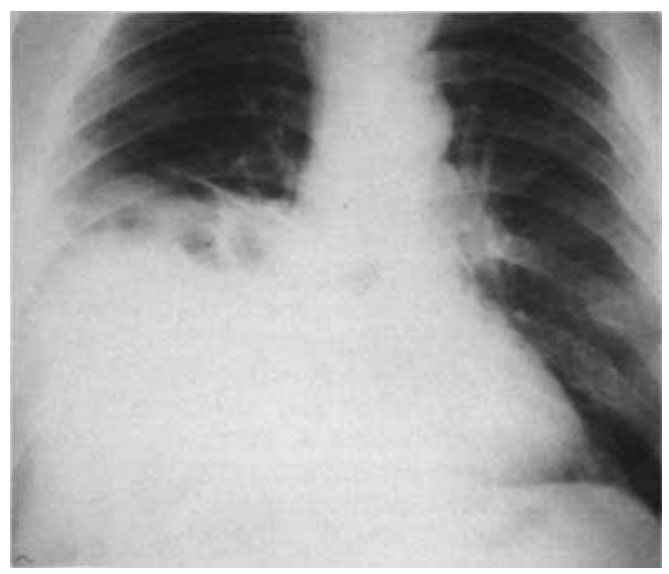

FIGURE 2A. - Case 2: PA view of the chest showing several lucencies and linear densities in the right lower lung field. The contour of the right hemidiaphragm is not clearly discernable. Bilateral rib fractures are noted. 


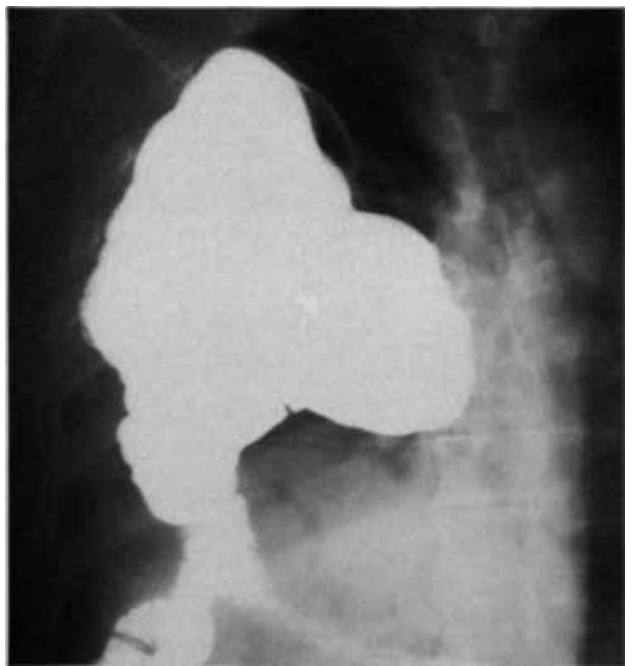

FIGURE 2B. - Case 2: A barium enema study showing herniation of cecum, ascending colon, proximal transverse colon and terminal ileum into the thorax through a defect in the dome of the right hemidiaphragm. An anomaly of fixation of the cecum was probably present prior to development of the diaphragmatic hernia. At surgery, part of the right lobe of the liver was also herniated into the thorax.

were heard over the right lower lung fields. The vital signs and remaining physical examination were within normal limits. The laboratory data, consisting of hematological and biochemical profile, urinalysis and sputum cytology were within normal limits.

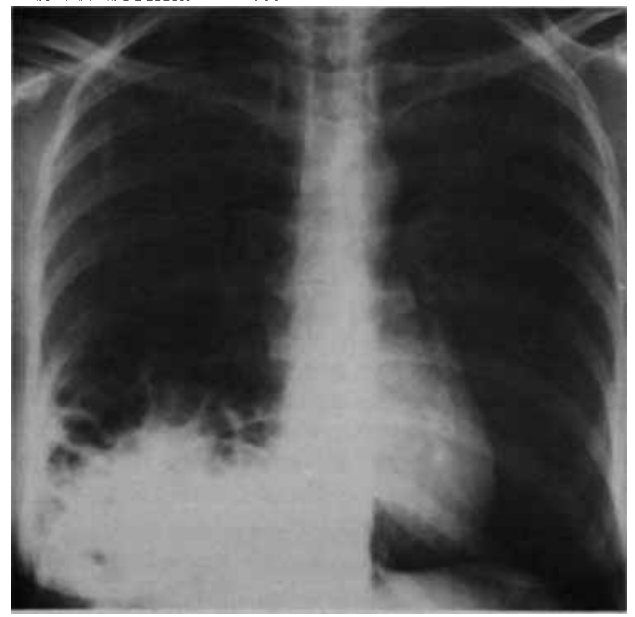

FIGURE 3A. - Case 3: PA view of the chest snowing several lucencies in the right lower thorax suggestive of bowel herniation.
Radiography of the chest (Figure 2A) revealed multiple cystic densities in the right lower hemithorax with several areas of linear compression atelectasis. The right hemidiaphragm was markedly elevated. Several old healed rib fractures were noted bilaterally. Bronchoscopy was unremarkable. Upper gastrointestinal examination was normal. However, the barium enema (Figure 2B) revealed a large diaphragmatic hernia containing caecum, ascending and proximal transverse colon. Part of the terminal ileum was also located within the thorax. Because of the symptoms of dyspnea and periodic abdominal pain and nausea in the presence of a large diaphragmatic hernia, intermittent obstruction of the bowel was suspected. At surgery, in addition to bowel, part of the right lobe of the liver was also found herniated into the thorax through a $6 \mathrm{~cm}$. defect in the dome of the right diaphragm. The bowel and liver were reduced into the abdomen and the diaphragmatic defect was closed. The patient had an uneventful postoperative course.

Comment: In most congenital diaphragmatic hernias the cecum is usually in its normal anatomic position. However, in this case it was intrathoracic. Therefore, it is believed that an anomaly of fixation of cecum may have been present prior to development of a traumatic diaphragmatic hernia.

\section{Case 3:}

A 36-year old white female was admitted to a local community hospital in December 1970 with a chronic history of dyspepsia and food sticking in the stomach for many hours after eating. She gave a history of an auto accident 15 years before in which she sustained several contusions and was hospitalized for one week. The chest radiograph at discharge was believed to be normal.

The physical examination was unremarkable except for percussion dullness and absent breath sounds over the right lower hemithorax. The laboratory data were within normal limits. A chest radiograph (Figure $3 \mathrm{~A}$ ) revealed several cystic lucencies in the right lower hemithorax suggesting bowel herniation. The right diaphragm could not be clearly discerned. An upper gastrointestinal and small bowel examination revealed large diaphragmatic hernia containing several loops of small bowel (Figure 3B).

Since the patient had occasional dyspnoea and chronic symptoms of vague abdominal pain at times accompanied by nausea and vomiting, it was believed that those symptoms were related 


\section{TRANSDIAPHRAGMATIC LIVER HERNIA IN ADULTS}

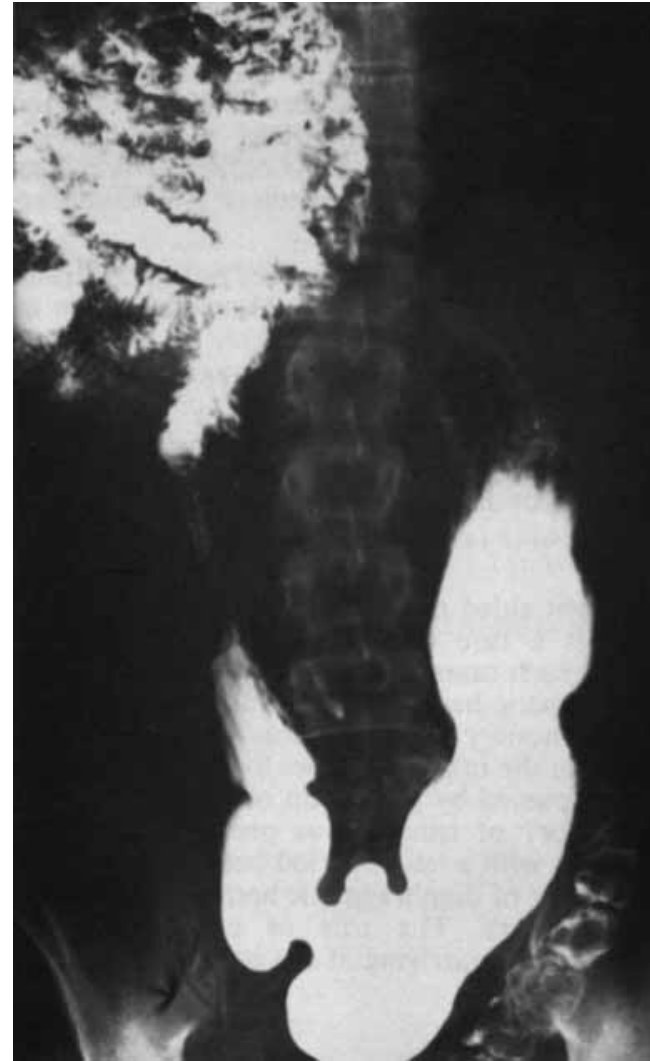

FIGURE 3B. - Case 3: An upper gastrointestinal and small bowel barium study confirming herniation of several loops of small bowel. At surgery a part of the right lobe of the liver was also herniated into the thorax.

to intermittent obstruction of bowel. The patient was taken to surgery. At operation a $4 \mathrm{~cm}$. defect was noted in the dome of the right diaphragm through which, in addition to loops of small bowel, a portion of right lobe of the liver was also herniated into the thorax. The herniated bowel and liver were reduced into the abdomen and the diaphragmatic defect was repaired. The patient had an uneventful post-operative course and has remained symptom free since her surgery.

\section{DISCUSSION}

Traumatic diaphragmatic hernias constitute only $5 \%$ of all diaphragmatic hernias ${ }^{11}$. The hernias may occur through any part of the diaphragm. Most reports indicate that left sided traumatic diaphragmatic hernias are far more common than those involving the right hemidiaphragm with relative incidences in the range of $95 \%$ and $5 \%$ respectively ${ }^{7}$. Childress and Grimes ${ }^{12}$ postulated that the right lobe of the liver and right kidney protect the right hemidiaphragm far better than the less bulky stomach, left lobe of liver, spleen and left kidney buffer the left hemidiaphragm, thus explaining the low incidence of right sided traumatic diaphragmatic hernia. The most common contents of a traumatic diaphragmatic hernia are stomach, large and small bowel. The liver may rarely herniate. More recent publications have shown an increased incidence of right sided traumatic diaphragmatic rupture ${ }^{13}$.

The first case of hepatic herniation mistaken for intra-thoracic tumor was described by Elder and Postlethwaiste in $1908^{14}$. The diagnosis was established at autopsy. Lilianthal, 1931'5, reported a middle-aged female complaining of heaviness in the chest with slight dyspnea and mild cough. The chest radiograph showed a large rounded mass in the right lower hemithorax thought to be a mediastinal dermoid. At surgery this mass was found to be the right lobe of the liver herniating through a defet in the diaphragm.

Harrington and Kirklin in $1938^{16}$ reviewed 131 cases of diaphragmatic hernias and stated: "In the rare case of hernia through the right arch of the diaphragma portion of the liver projects through the breech and is likely mistaken for a neoplasm". Harrington ${ }^{17}$ also reported upon 304 patients with diaphragmatic hernia who had surgical repair. Only one hernia occurred on the right side and contained liver, stomach, duodenum, small bowel and the head of the pancreas.

In 1945 Wagner $^{1}$ reported a case in which a portion of the liver, the size of a hen's egg, herniated through the right cardiophrenic angle of the diaphragm.

Wolfson and Goldman ${ }^{18}, 1948$, reported a 47 year old female complaining of attacks of epigastric pain, nausea, and vomiting during the preceding six months. A mass lesion in the right lower hemi-thorax was found on chest radiography. At surgery a well defined, vestigeal, mesentery-like transparent veil covered the herniated liver protruding through the diaphragmatic defect. It was thought that the veil was a congenital diaphragmatic anomaly and that the liver hernia was also probably congenital, remaining asymptomatic until periodic strangulation occurred. 


\section{F.P. AGHA}

Peck $^{9}$, in a review of 23 post traumatic diaphragmatic hernias involving the liver classified liver herniation into three types:

Type 1: Isolated total liver herniation

Type II: Isolated partial liver herniation

Type III: Liver herniation associated with other abdominal viscera.

In type I, the entire liver herniates into the thorax producing a high smooth acurate shadow giving the impression of an elevated right hemidiaphragm or eventration ${ }^{7}$. The type II hernia involves only a portion of the liver and causes a typical mushroom projection into the thorax. The Juxtadiaphragmatic smooth rounded mass of liver may be mistaken for a pulmonary, pleural, mediastinal or diaphragmatic tumor ${ }^{8}, 19$. In the type III hernia, involving other portions of the gastrointestinal tract in addition to liver, roentgenographic examination usually demonstrates intrathoracic loops of bowel and varying degrees of pleuro-pulmonary changes. One type of liver herniation may change to another type over a period of time or after recurrence of trauma ${ }^{13}$.

A preoperative diagnosis of chronic right sided traumatic diaphragmatic hernia is not made in most cases. As many as $60 \%$ of these patients have vague, non-specific chest or epigastric pain when an abnormality of their chest roentgenogram is discovered. Some experience dyspnea, particularly upon deep inspiration. The gastrointestinal symptoms which occur when either large or small bowel is involved in the hernia are not unlike those which have been described for incarcerated obstructed left diaphragmatic hernia.

A prompt diagnosis of diaphragmatic herniation is the exception and not the rule with an average interval between injury and diagnosis of 3 or 4 years on the left side and 9 years on the right side ${ }^{7}$. Furthermore, after initial symptoms of trauma subside, there is frequently a latent asymptomatic period varying from 3 months to 32 years $^{9}$ with several reported cases of repair $30-44$ years after initial injury 10 . This latent period had been also emphasized in the more common left sided traumatic diaphragmatic hernia by Carter et $a l^{s}$.

In the evaluation of Juxta-diaphragmatic mass lesions radionuclide liver scan should be performed in all cases 20,21 . The CT scan can provide similar information regarding the presence of supra-diaphragmatic hepatic tissue by virtue of its attenuation coefficient. However, ultrasound can quite clearly demonstrate the dia- phragmatic defect not demonstrable by radionuclide liver scan or CT scan. The utilization of ultrasonography in the evaluation of Juxtadiaphragmatic mass lesions will mitigate the use of more invasive procedures like diagnostic pneumoperitoneum and angiography ${ }^{22}$ previously advocated in the evaluation of transdiaphragmatic liver hernias.

In summary, the diagnosis of right sided transdiaphragmatic hepatic herniation rests upon an awareness of this entity, a recent or old history of trauma and Juxta-diaphragmatic abnormalities. Utilization of current noninvasive imaging modalities and in particular radionuclide liver scan, CT scan, and ultrasound can establish the correct diagnosis in almost all cases.

\section{ABSTRACT}

Right sided transdiaphragmatic liver herniation is a rare type of diaphragmatic hernia. Three such cases in adults are reported. A localized hepatic herniation (type III) masquerading as pulmonary neoplasm was revealed in one, while in the other two cases liver herniation was accompanied by herniation of bowel (type III). A history of trauma was present in all three patients with a latent period between injury and discovery of diaphragmatic hernia ranging from 10-20 years. The role of current imaging modalities in arriving at the correct diagnosis is emphasized.

\section{REFERENCES}

1. Wagner A. Four cases of diaphragmatic intumescence. Acta Radiol 1945; 26 : 239-247.

2. Hedblom CA. Diaphragmatic hernia. Ann Int Med $1934 ; 8: 156-176$

3. Arnheim EE. Congenital hernia of the diaphragm with special reference to right sided hernia of the liver and intestines. Surg Gynecol Obstet 1952; 95 : 293-307.

4. Ban JL and Moore TC. Intrathoracic tension incarceration of stomach and liver through right sided congenital posterolateral diaphragmatic hernia. J Thoracic Cardio Vasc Surg 1973; 66 : 969-973.

5. Korobkin MT, Miller SW, DeLorimier AA, deLorimier AA, Gordon $S$ and Palubinskas AJ. Hepatic herniation through the Morgagni foramen. Amer J Dis Child 1973; 126 : 217-219.

6. Poe RH and Schowegerdt CG. Two cases of a traumatic herniation of the liver. Amer Rev Resp Dis 1972; 105 : 959-963.

7. Orringer MB, Kirsh MM and Sloan H. Congenital and traumatic diaphragmatic hernias exclusive of the hiatus. Current Prob Surg 1975; March : $1-64$.

8. Carter BN, Guiseffi J and Felson B. Traumatic diaphragmatic hernia. Amer J Roentgenol 1951; 65 : 56-72. 


\section{TRANSDIAPHRAGMATIC LIVER HERNIA IN ADULTS}

9. Peck Jr WA. Right-sided diaphragmatic liver hernia following trauma. Amer J Roentgenol 1957; 78 : 99-108.

10. Child CG, Harmon GS, Potter CT and Steinber IJ. Liver herniation simulating intrathoracic tumor. J Thoracic Cardiovase Surg 1951; $21: 391-393$.

11. Ghahremani GG and Meyers MA. Herniation of the colon in Greenbaum E 1 (Ed). Radiographic Atlas of Colon Disease -- Yearbook Medical Publisher Inc. Chicago Illinois 1980; 215-231.

12. Childress ME and Griems OF. Immediate and remote sequalae in traumatic diaphragmatic hernias. Surg Gynecol Obstet 1961; $113: 573-584$.

13. Estrera AS, Platt MR, Mills LJ and Urschel HC. Rupture of the right hemidiaphragm in the liver herniation: Report of a case with extenstion of a tear of a previously undiagnosed ruptured right hemidiaphragm. J Trauma 1980; $20: 174-176$.

14. Elder EA and Postlethwaite JM. Note on abnormality of the liver simulating thoracic tumor. Lancet $1908 ; 1$ $: 1073$.

15. Lilienthal H. Diaphragmatic hernia of the liver. Surg Clin NA 1931; $11: 475-481$.
16. Harrington SW and Kirklin BR. Clinical and roentgenologic manifestations and surgical treatment of diaphragmatic hernia with a review of 131 cases. Radiology 1938; $30: 147-156$.

17. Harrington SW. Diaphragmatic hernia of children. Ann Surg 1942; 115 : 705-715.

18. Wolfson SA and Goldman A. Strangulating diaphragmatic hernia of the liver. Surgery 1948; 24 : 846-852.

19. Fanta GH, Kacoyanis GP, Koster JR and Mcfadder ER. Pseudo-pseudo tumor of the lung: Hepatic herniation into the right major fissure imitating a pseudo tumor on chest roentgenogram. Chest $1980 ; 78$ : 346-348.

20. Cheung D and Meakins JL. Diagnosis of right sided diaphragmatic rupture by liver lung scanning. Canadian J Surg 1980; 23 : 302-303.

21. Harman PK, Mentzer RM, Winberg AC, Caldwell PC and Minor GR. Early diagnosis by liver scan of a right sided traumatic diaphragmatic hernia. J Trauma $1981 ; 21: 489-490$.

22. Enge $I$ and Froysaker T. Rupture of the right diaphragm in the herniation of liver. Radiology 1964; 92 : 1273-1274. 